\title{
BMJ Open Is there a role for physical activity when treating patients with cancer with immune checkpoint inhibitors? Protocol for a scoping review
}

\author{
Miaoqi Chen, ${ }^{1}$ Ridesh Rai, ${ }^{1}$ Louis Fox (D) , ${ }^{1}$ Charlotte Louise Moss (D) , ${ }^{1}$ \\ Gincy George, ${ }^{1}$ Sophia N Karagiannis, ${ }^{2,3}$ Deborah Enting, ${ }^{1,4}$ Magdalene Joseph, ${ }^{5}$ \\ Nicola Peat, ${ }^{6}$ Beth Russell (D) , ${ }^{1}$ Mieke Van Hemelrijck (D) ${ }^{1}$
}

To cite: Chen M, Rai R, Fox L, et al. Is there a role for physical activity when treating patients with cancer with immune checkpoint inhibitors? Protocol for a scoping review. BMJ Open 2021;11:e046052. doi:10.1136/ bmjopen-2020-046052

- Prepublication history and additional supplemental material for this paper are available online. To view these files, please visit the journal online. (http://dx.doi.org/10.1136/ bmjopen-2020-046052)

$\mathrm{MC}$ and RR are joint first authors.

$\mathrm{BR}$ and MVH are joint senior authors.

Received 26 April 2021 Accepted 11 August 2021
Check for updates

(C) Author(s) (or their employer(s)) 2021. Re-use permitted under CC BY-NC. No commercial re-use. See rights and permissions. Published by BMJ.

For numbered affiliations see end of article.

Correspondence to

Beth Russell;

beth.russell@kcl.ac.uk

\section{ABSTRACT}

Introduction For patients with cancer, immune checkpoint inhibitors (ICls) produce superior long-term responses compared with alternative treatments, although at the cost of manifesting adverse immune-related events. There are many hypotheses of the impacts of physical activities in immunotherapy, but little is known about the oncological outcomes and the underlying mechanisms.

This scoping review aims to identify possible physical activity interventions, their efficacy and feasibility and the potential underlying biological mechanisms responsible for their effects.

Method and analysis The Levac methodology framework was used along with guidance from the Joanna Briggs Institute Manual for Evidence Synthesis to inform development of this protocol. Abstracts and titles followed by full-text screening will be performed by two independent reviewers for inclusion. All studies describing the impact of physical activities and exercise interventions on cancer ICls, with particular focus on oncological outcomes, quality of life or underling biological mechanisms, will be included. After extracting qualitative and quantitative data, they will be evaluated and summarised, respectively. Subsequently, a further consultation step with other scientists and healthcare professionals will be performed.

Ethics and dissemination The research findings will be published through an open-access peer-reviewed journal. The results of this scoping review will be used to inform further studies on physical impacts on immunotherapy. All data included will be from open resources, therefore, no ethical clearances are required.

\section{INTRODUCTION}

As a leading cause of death in the UK, cancer accounts for nearly a quarter of the total deaths between 2015 and 2017: around 367000 new cancer cases occur in the UK every year, and almost 1000 cases every day. ${ }^{1}$ It is well established that engagement with physical activity (PA) (as defined by WHO as any bodily movement produced by skeletal muscles that requires energy expenditure ${ }^{2}$ ),
Strengths and limitations of this study

- Use of multiple databases to source literature.

- Inclusion of consultation phase to include the viewpoint of healthcare professionals and scientists.

- Quantitative analysis only possible if sufficient data are available

is beneficial to patients diagnosed with cancer. ${ }^{3}$ Suggested benefits of PA include improvements to cancer-specific quality of life (QoL), improvements to cancer-specific fatigue and amelioration of certain treatment side effects such as pain, weakness and anxiety. However, a new line of investigation has emerged aiming to address whether the benefit of PA for patients with cancer extends beyond symptom control. ${ }^{4-6}$ For example, preclinical mouse melanoma models have shown that PA has an additive effect in controlling tumour progression in conjunction with programmed death-1 receptor (PD-1) blocking antibodies, ${ }^{78}$ without a pharmacological side effect profile.

With the recent advances in immunotherapy for cancer (ie, the development of various immune checkpoint inhibitors (ICIs)), it is also of interest to note that preclinical studies have shown that PA may increase the number of dendritic cells. ${ }^{4}$ Immunotherapy with monoclonal antibodies targeting cytotoxic T lymphocyte-associated antigen 4 and PD-1 and its ligand- 1 has become standard of care for an increasing number of cancer types, but come with a range of infusion reactions and both general and immune-related adverse events. ${ }^{5}$ Despite ICIs being increasingly used to treat a variety of cancers, comparatively little is known about how to balance their response and adverse events. We hypothesise 
here than PA could be instrumental in this this balance. Since ICIs can achieve long-term responses superior to those seen with most other treatments, but only works in subsets of patients, treatments combined with alternative strategies that enhance responses are an important area of research.

\section{Study rationale}

PA is likely to have benefits in combination with cancer treatment with limited side effects and should therefore be explored further. ${ }^{3}$ Thus, since ICIs are leading the way in the treatment of various cancers, the current scoping review specifically focuses on identifying what is already known about PA interventions in this immunotherapy setting $^{910}$ and where the current gaps are to advance current research.

\section{Study objectives}

In this scoping review, we aim to investigate the current evidence and gaps in our understanding of the effects of PA on ICIs for cancer, with a specific focus on: (1) oncological outcomes; (2) QoL and (3) potential underlying biological mechanisms.

\section{METHODS}

\section{Protocol design}

The Joanna Briggs Institute Manual for Evidence Synthesis was used as reference during the development of this protocol. ${ }^{11}$ The overall protocol framework also closely resembles the methodological guidelines developed by Levac et al. ${ }^{12}$ The protocol is also registered on https://osfio/.

\section{Stage 1: identifying the research question}

Following consultation with the clinical research team, the proposed research questions are defined as:

1. Is there any evidence that suggests that PA has a demonstrable effect on improving the oncological outcomes of patients with cancer receiving ICIs?

2. Is there any evidence that suggests that PA (including which type, timing and dosage of PA) has a demonstrable effect on improving the QoL of patients with cancer receiving ICIs?

3. What are the biological mechanisms, if any, that could be responsible for the effects exerted by PA on improving the oncological outcomes and QoL of patients with cancer receiving ICIs?

\section{Stage 2: identifying relevant studies: search strategy}

The following electronic databases will be searched from inception until the date on which the searches will be performed: MEDLINE, Embase and PsycINFO. Search terms have been determined through researcher input and researching the current available literature to help guide the selection of terms-ensuring they are broad enough to capture the three research questions outline above. The search strategy can be found in online supplemental appendix.
Stage 3: study selection

Studies will be considered for inclusion if they assess oncological outcomes or QoL related to PA in patients who receive ICIs for their cancer, or if they assess potential underlying biological mechanisms for a link between PA and biological processes involved in immunotherapy treatment. Hence, we will include observational studies, randomised controlled trials (RCTs) and preclinical studies. We understand that there will be strengths and limitations to all types of studies and these will be discussed more within the results of the scoping review. Studies will be excluded if the publication is not available in English. All papers derived from the digital search process will be uploaded to Excel using a reference management software (EndNote). From these references, we will then document the exclusion process of the studies; initially excluding irrelevant studies based on title alone, then based on abstracts. Two review authors will screen the studies independently. Any lack of consensus will be discussed with a third review author. After screening titles and abstracts, the full articles will be read and considered for the review; those excluded will have recorded evidence as to why this was necessary. For studies that have multiple publications of the same outcome(s) reported, the one with the longest follow-up will be included.

\section{Stage 4: charting the data}

Two independent reviewers will conduct this process. The data extraction table produced will include at least the following headings:

- Author(s).

- Year of publication.

- Origin/country of origin (where the study was published or conducted).

- Aims/purpose.

- Study population.

- Study design.

- PA type +details (eg, lifestyle, aerobic, strength, timings, dosage).

- Supervised/unsupervised PA.

- Study aims and outcomes investigated (eg, oncological outcome, QoL, biological mechanism).

- Key findings that relate to scoping review objectives (including any assessment of feasibility of intervention).

\section{Stage 5: collating, summarising and reporting the results}

As per the methodological guidelines developed by Levac et al, this stage will consist of three distinct steps: analysing the data, reporting results and applying meanings to the results. ${ }^{11}$

For the first two research aims, where possible, we will perform a quantitative analysis and evaluate both measurements for oncological outcomes (eg, 5-year survival rates, recurrence, response rates) and QoL (eg, validated questionnaires). In addition, we will describe the current research in terms of evidence available (eg, RCTs vs observational data). For the third research question, we will provide a summary of the extent to which current 
findings support a biological link between PA and immunotherapy outcomes, and if so what the content of these findings are. The studies will be compared in a narrative manner. Findings will be reported in accordance with the Preferred Reporting Items for Systematic Reviews and Meta-Analyses extension for Scoping Reviews. ${ }^{13}$

\section{Stage 6: consultation}

This scoping review is a first phase in a multistage research programme aimed at developing a feasibility PA intervention for patients with cancer on ICIs. To ensure that our assessment of the existing evidence for the potential role of PA in cancer ICIs, we also aim to include a consultation phase in this scoping review. The results from this scoping review combined with the consultation phase will then lead to development of a strategy for further research into this fieldfor example, an RCT based on an exercise intervention for patients with cancer on ICIs with a translational component to investigate underlying biological mechanisms.

This consultation phase is part of our patient and public involvement (PPI) strategy as we will work actively in partnership with patients, healthcare professionals and scientists to better understand how we can design future research into PA and ICIs. ${ }^{14}$ More specifically, we will run a focus group with patient representatives, immunologists, healthcare professionals, physiotherapists and epidemiologists to help identify whether the results of the scoping review truly reflect the gaps in current research.

\section{Patient and public involvement}

Patients, healthcare professionals and scientists will be involved during the consultation phase (stage 6) of the study.

\section{Dissemination and ethics}

As outlined above, this scoping review with a consultation phase will constitute the first stage in a multistage research programme aimed at developing a PA intervention for patients with cancer on ICIs.

As the scoping review methodology is based on reviewing and collecting data from publicly available materials, this study does not require ethics approval. The consultation stage will be carried out as part of PPI, so does not require ethical approval either. To facilitate knowledge translation activities, we will liaise with relevant stakeholders through patient advocate and healthcare professional organisations.

\footnotetext{
Author affiliations

${ }^{1}$ Translational Oncology \& Urology Research, School of Cancer \& Pharmaceutical

Sciences, King's College London, London, UK

${ }^{2}$ St John's Institute of Dermatology, School of Basic \& Medical Biosciences, King's College London, London, UK

${ }^{3}$ Breast Cancer Now Research Unit, School of Cancer \& Pharmaceutical Sciences, King's College London, London, UK

${ }^{4}$ Department of Medical Oncology, Guy's and Saint Thomas' NHS Foundation Trust, London, UK

${ }^{5}$ Immunobiology, School of Immunology \& Microbial Sciences, King's College

London, London, UK

${ }^{6}$ Guy's and St Thomas' NHS Foundation Trust, London, UK
}

Correction notice This article has been corrected since it first published. Author name 'Ridesh Rai' has been updated.
Twitter Louis Fox @_LouisFox and Sophia N Karagiannis @SophiaKaragiann

Contributors Study concept, design and planning: MC, RR, LF, GG, SK, DE, MJ, NP, BR and MVH. Protocol writing and review: CLM, RR, LF, CLM, GG, SK, DE, MJ, NP, BR and MVH.

Funding The authors have not declared a specific grant for this research from any funding agency in the public, commercial or not-for-profit sectors.

Competing interests None declared.

Patient consent for publication Not required.

Provenance and peer review Not commissioned; externally peer reviewed.

Supplemental material This content has been supplied by the author(s). It has not been vetted by BMJ Publishing Group Limited (BMJ) and may not have been peer-reviewed. Any opinions or recommendations discussed are solely those of the author(s) and are not endorsed by BMJ. BMJ disclaims all liability and responsibility arising from any reliance placed on the content. Where the content includes any translated material, BMJ does not warrant the accuracy and reliability of the translations (including but not limited to local regulations, clinical guidelines, terminology, drug names and drug dosages), and is not responsible for any error and/or omissions arising from translation and adaptation or otherwise.

Open access This is an open access article distributed in accordance with the Creative Commons Attribution Non Commercial (CC BY-NC 4.0) license, which permits others to distribute, remix, adapt, build upon this work non-commercially, and license their derivative works on different terms, provided the original work is properly cited, appropriate credit is given, any changes made indicated, and the use is non-commercial. See: http://creativecommons.org/licenses/by-nc/4.0/.

\section{ORCID iDs}

Louis Fox http://orcid.org/0000-0002-8280-1797

Charlotte Louise Moss http://orcid.org/0000-0002-4354-8987

Beth Russell http://orcid.org/0000-0001-5640-8425

Mieke Van Hemelrijck http://orcid.org/0000-0002-7317-0858

\section{REFERENCES}

1 Cancer Research UK. Cancer Incidence Statistics [Internet], 2020. Available: https://www.cancerresearchuk.org/health-professional/ cancer-statistics/incidence [Accessed 12 Oct 2020].

2 World Health Organisation. Physical Activity [Internet], 2020. Available: https://www.who.int/news-room/fact-sheets/detail/ physical-activity

3 Koelwyn GJ, Wennerberg E, Demaria S, et al. Exercise in regulation of Inflammation-Immune axis function in cancer initiation and progression. Oncology 2015;29:908-22.

4 Fox L, Cahill F, Burgess C, et al. Real world evidence: a quantitative and qualitative glance at participant feedback from a Free-Response survey investigating experiences of a structured exercise intervention for men with prostate cancer. Biomed Res Int 2017;2017:1-10.

5 Sheill G, Brady L, Guinan E, et al. The expect (examining exercise, prostate cancer and circulating tumour cells) trial: study protocol for a randomised controlled trial. Trials 2017;18:456.

6 Christensen J, Simonsen C, Hojman P. Exercise training in cancer control and treatment. Compr Physiol 2020;9:165-205.

7 Idorn M, thor Straten P. Exercise and cancer: from "healthy" to "therapeutic"? Cancer Immunology, Immunotherapy 2017;66:667-71.

8 Wennerberg E, Lhuillier C, Rybstein MD, et al. Exercise reduces immune suppression and breast cancer progression in a preclinical model. Oncotarget 2020;11:452-61.

9 Belcher D, Enting D, Fox L. Characteristics of bladder cancer patients underoing immunotherapy - are they a suitable population for an exercise intervention programme? Second Bladder Cancer Translational Research Meeting. Birmingham: Frontiers in Oncology, 2020.

10 Joseph M, Enting D. Immune responses in bladder Cancer-Role of immune cell populations, prognostic factors and therapeutic implications. Front Oncol 2019;9:1270.

11 Aromataris E, Munn Z. JBI manual for evidence synthesis, 2020.

12 Levac D, Colquhoun H, O'Brien KK, O'Brien K. Scoping studies: advancing the methodology. Implement Sci 2010;5:69.

13 Tricco AC, Lillie E, Zarin W, et al. PRISMA extension for scoping reviews (PRISMA-ScR): checklist and explanation. Ann Intern Med 2018;169:467-73.

14 Baxter S, Clowes M, Muir D, et al. Supporting public involvement in interview and other panels: a systematic review. Health Expect 2017;20:807-17. 